\title{
MULTIPLE SOLUTIONS OF A DIRICHLET PROBLEM IN ONE-DIMENSIONAL BILLIARD SPACE
}

\author{
JAN TOMEČEK
}

Received 19 September, 2017

Abstract. The paper gives multiplicity results for the impulsive boundary value problem

$$
\begin{gathered}
x^{\prime \prime}=f(t, x), \quad \text { for a.e. } t \in[0, T], \text { such that } x(t) \in \operatorname{int} K, \\
x^{\prime}(s+)=-x^{\prime}(s-), \quad \text { if } s \in(0, T), x(s) \in \partial K, \\
x(0)=A, \quad x(T)=B,
\end{gathered}
$$

where $K \subset \mathbb{R}$ is a compact interal, $f$ is Carathéodory function on $[0, T] \times K$ and $A, B \in$ int $K$. This problem can be understood as a problem in one-dimensional billiard space and it is also a generalization of oscillator with obstacles from below and from above and absolutely elastic impacts. A simple condition for the existence of solution with exact number of impacts is given, as well as the multiplicity result. The results are obtained by a transformation into problem without impulses (without impacts) and using Schauder fixed point theorem.

2010 Mathematics Subject Classification: 34A37; 34B37

Keywords: state-dependent impulses, system with impact, billiard space, Dirichlet problem, existence and multiplicity result

\section{INTRODUCTION}

The theory of impulsive differential equations is quite well developed theory. It received a lot of attention during the last decades, see e.g. monographs $[1,3,5,6,8$, 10,11]. The theory enables to model phenomena described by differential equations where abrupt changes on short time intervals (often caused by external forces) occur. It is more advantageous to model these changes as instantaneous (so called impulses).

Systems with impact draw attention of many mathematicians and physicists in the past several centuries. Let us mention a monograph [7] and some papers investigating periodic solutions of linear impact oscillator, see e.g. [2,9] and references therein. Note, that the dynamics with impacts can be understood by means of impulsive differential equations. Here the impulse (discontinuity) occurs at the time of impact and the velocity (derivative) undergoes discontinuous change (the mass bounces off of an obstacle), e.g. billiard ball hits the boundary of the table, mass on spring hits some obstacle, etc. 
Recently, in [4], Dirichlet problem in billiard spaces with absolutely elastic impact

$$
\begin{aligned}
& x^{\prime \prime}=f(t, x) \quad \text { for a.e. } t \in[0, T], x(t) \in \operatorname{int} K, \\
& \triangle x^{\prime}(s)=I\left(x(s), x^{\prime}(s)\right), \quad x(s) \in \partial K, \\
& x(0)=x(T)=0,
\end{aligned}
$$

was investigated for two cases: (i) one-dimensional case, i.e. $K \subset \mathbb{R}$ is a compact interval, (ii) multidimensional case, for $K \subset \mathbb{R}^{2 k}, k \in \mathbb{N}$, certain appropriate compact set. In the one-dimensional case, there were reached multiplicity results under conditions that $f$ is Carathéodory condition and is Lipschitz continuous in the second variable. The existence of infinitely solutions are then guaranteed. The purpose of this paper was to give more general and more detailed information than in [4] for the one-dimensional case. Here, the Lipschitz condition is not needed. Moreover, the exact number of impacts is given (see Theorem 1). The reason of the weakened assumptions lies in the fact that paper [4] relies on the shooting method and continuous dependence on initial values of solution. On the other hand, the idea in [4] can be quite easily generalized for higher dimensions. Here, the idea is to investigate an auxiliary problem without impulses.

In this paper the impulsive boundary value problem

$$
\begin{gathered}
x^{\prime \prime}=f(t, x), \quad \text { for a.e. } t \in[0, T], \text { such that } x(t) \in \operatorname{int} K, \\
x^{\prime}(s+)=-x^{\prime}(s-), \quad \text { if } s \in(0, T), x(s) \in \partial K, \\
x(0)=A, \quad x(T)=B,
\end{gathered}
$$

where $K \subset \mathbb{R}$ is some compact interval, $f:[0, T] \times K \rightarrow \mathbb{R}$ is a Carathéodory function, $A, B \in$ int $K$, is investigated. The impulsive condition (1.2) represents absolutely elastic impact on the boundary of $K$.

First let us give the definition of a solution to this problem.

Definition 1. The function $x \in \mathrm{C}([0, T])$ is called a solution of the problem (1.1),(1.2), if and only if

- there exists a finite set $\left\{t_{1}, \ldots, t_{p}\right\} \subset(0, T), t_{1}<t_{2}<\ldots<t_{p}$ such that $\left.x\right|_{\left[t_{i}, t_{i+1}\right]} \in \mathrm{AC}^{1}\left(\left[t_{i}, t_{i+1}\right]\right)$ for $i=0, \ldots, p$, where we put $t_{0}=0, t_{p+1}=T$,

- $x(t) \in K$ for each $t \in[0, T]$ and $x(t) \in \partial K$ iff $t=t_{i}$ for some $i=1, \ldots, p$,

- $x$ satisfies the differential equation (1.1) and impulsive conditions (1.2).

The number $p$ is called a number of impacts of a solution $x$. A solution $x$ of $(1.1),(1.2)$ is called a solution of (1.1)-(1.3), if it satisfies (1.3).

The main result of this paper is the following.

Theorem 1. Let $K=[a, b]$ be a compact interval, $f \in \operatorname{Car}([0, T] \times K)$ and $A, B \in$ int $K$. Then for each $p \in \mathbb{N}$ satisfying

$$
p>\frac{T}{b-a} \int_{0}^{T}|m(t)| \mathrm{d} t+1,
$$


where

$$
m(t)=\max _{x \in K}|f(t, x)|, \quad \text { a.e. } t \in[0, T],
$$

the problem (1.1)-(1.3) has at least two solutions with exactly $p$ impacts.

The paper is organized as follows. In Section 2 necessary notation and some trivial results are presented. In Section 3, auxiliary singular nonimpulsive problem is investigated and its relation to the original problem is shown. Finally, Section 4 contains the main results of the paper, incl. the proof of Theorem 1.

\section{NOTATION AND PRELIMINARIES}

By $\mathbb{N}, \mathbb{Z}, \mathbb{R}$ we denote the set of all positive integers, integers and reals, respectively. By $\mathrm{AC}(J),\left(\mathrm{AC}^{1}(J)\right)$ we denote the set of absolutely continuous functions on the interval $J$ (having absolutely continuous derivatives on $J$ ) and by $\mathrm{C}([0, T]$ ), $\mathrm{C}^{1}([0, T])$ the space of continuous functions on the interval $[0, T]$ (having continuous derivatives) equipped with standard norms. Moreover, $\mathrm{L}^{1}([0, T])$ is the space of all Lebesgue integrable functions on $[0, T]$ with the norm $\|x\|_{\mathrm{L}^{1}}=\int_{0}^{T}|x(t)| \mathrm{d} t$, $x \in \mathrm{L}^{1}([0, T])$. By $\operatorname{Car}([0, T] \times \Omega)$, where $\Omega \subset \mathbb{R}$ we mean the set of all functions $f:[0, T] \times \Omega \rightarrow \mathbb{R}$ satisfying Carathéodory conditions:

- for each $x \in \Omega$ the function $f(\cdot, x)$ is Lebesgue measurable on $[0, T]$,

- for a.e. $t \in[0, T]$ the function $f(t, \cdot)$ is continuous on $\Omega$,

- for each compact set $K \subset \Omega$, there exists $m \in \mathrm{L}^{1}([0, T])$ such that $|f(t, x)| \leq$ $m(t)$ for a.e. $t \in[0, T]$.

Also, let us remind some facts about the fractional part of a real number: For $x, a \in \mathbb{R}$, $a>0$ we denote

$$
x \bmod a:=x-a\left\lfloor\frac{x}{a}\right\rfloor,
$$

where $\lfloor x\rfloor$ stands for the integer part of $x$. Let us note that the function

$$
\mathbb{R} \ni t \mapsto t \bmod a
$$

is $a$-periodic function.

Remark 1. Let $x: J \rightarrow \mathbb{R}, J \subset \mathbb{R}$ be an interval, $a \in \mathbb{R}, a>0, x \bmod a \neq 0$ on $J$, $i \in \mathbb{N}$. Then

- if $x$ is continuous on $J$, then is $x \bmod a$;

- if $x$ has an $i$-th derivative at $t \in \operatorname{int} J$, then $(x \bmod a)^{(i)}(t)=x^{(i)}(t)$.

Lemma 1. Let $R \in \mathbb{R}, R>0$ and $\varphi:(0, R) \rightarrow \mathbb{R}$. Then the function $\varphi^{*}: \mathbb{R} \rightarrow \mathbb{R}$ defined by

$$
\varphi^{*}(x)= \begin{cases}\varphi(x \bmod 2 R) & \text { if } x \bmod 2 R \in(0, R), \\ -\varphi(2 R-(x \bmod 2 R)) & \text { if } x \bmod 2 R \in(R, 2 R), \\ 0 & \text { if } x \bmod R=0\end{cases}
$$


is $2 R$-periodic and odd function.

Proof. The periodicity follows directly from the definition of $\varphi^{*}$ and periodicity of the mapping $x \mapsto x \bmod 2 R$. In order to prove that the function is odd we consider three cases:

CASE A. Let $x \bmod R=0$. Then also $(-x) \bmod R=0$ and therefore $\varphi^{*}(-x)=0=$ $-\varphi^{*}(x)$.

CASE B. Let $x \bmod 2 R \in(0, R)$. Then there exists an integer $k$ such that $x=$ $2 R k+x \bmod 2 R$. Then

$$
-x=2 R(-k)-x \bmod 2 R=2 R(-k-1)+2 R-x \bmod 2 R,
$$

where $2 R-x \bmod 2 R \in(R, 2 R)$ and therefore $(-x) \bmod 2 R=2 R-x \bmod 2 R \in$ $(R, 2 R)$. Then

$$
\begin{gathered}
\varphi^{*}(-x)=-\varphi(2 R-(-x) \bmod 2 R)=-\varphi(2 R-(2 R-x \bmod 2 R)) \\
=-\varphi(x \bmod 2 R)=-\varphi^{*}(x) .
\end{gathered}
$$

CASE C. The case $x \bmod 2 R \in(R, 2 R)$ can be proved similarly to the previous one.

Remark 2. If $\varphi$ is continuous on $(0, R)$, then from the properties of $\varphi^{*}$ (from Lemma 1$)$ it follows only that $\varphi^{*}$ is continuous on each interval $(k R,(k+1) R)$, $k \in \mathbb{Z}$ and

$$
\varphi^{*}(k R+)=-\varphi^{*}(k R-)= \begin{cases}\varphi(0+) & \text { if } k \text { is even, } \\ -\varphi(R-) & \text { if } k \text { is odd. }\end{cases}
$$

Since $\varphi^{*}(k R)=0$ for $k \in \mathbb{Z}$, the function $\varphi^{*}$ has discontinuities at $x=k R, k \in \mathbb{Z}$ unless $\varphi(0+)=\varphi(R-)=0$.

Remark 3. In the next section will use function

$$
\Delta(t)= \begin{cases}t \bmod 2 R & \text { if } t \bmod 2 R \in[0, R), \\ 2 R-t \bmod 2 R & \text { if } t \bmod 2 R \in[R, 2 R) .\end{cases}
$$

Let us note that inclusion $t \bmod 2 R \in[0, R)$ is equivalent to $t \in[2 k R,(2 k+1) R)$ for some $k \in \mathbb{Z}$ and the inclusion $t \bmod 2 R \in[R, 2 R)$ is equivalent to $t \in[(2 k+$ 1) $R, 2(k+1) R)$ for some $k \in \mathbb{Z}$. Then, we can see that $\Delta: \mathbb{R} \rightarrow \mathbb{R}, \Delta(t)=|t|$ for $t \in[-R, R]$ and $\Delta$ is $2 R$-periodic. Therefore, range of $\Delta$ is $[0, R]$, it is a piecewise linear function, it is Lipschitz continuous on $\mathbb{R}$. Moreover,

$$
\Delta^{\prime}(t)=(-1)^{k} \quad \text { if } t \in(k R,(k+1) R), k \in \mathbb{Z} .
$$




\section{SingUlar AUXILIARY PROBLEM}

In this section we assume that

$$
R \in \mathbb{R}, R>0, K=[0, R], T>0, f \in \operatorname{Car}([0, T] \times K),
$$

and let us denote

$$
m(t):=\sup _{x \in K}|f(t, x)| \quad \text { for a.e. } t \in[0, T], \quad \bar{m}:=\|m\|_{\mathrm{L}^{1}} .
$$

Unlike the paper [4], here the existence result is obtained by using an appropriate "extension" of the right-hand side of the equation (1.1) onto the set $[0, T] \times \mathbb{R}$. Note that this extension is not neccessarily a Carathéodory function on $[0, T] \times \mathbb{R}$, but it has possible discontinuities in the state variable.

We define an auxiliary function $f^{*}:[0, T] \times \mathbb{R} \rightarrow \mathbb{R}$ by

$$
f^{*}(t, x)=\left\{\begin{array}{ll}
f(t, x \bmod 2 R) & \text { if } x \bmod 2 R \in(0, R), \\
-f(t, 2 R-(x \bmod 2 R)) & \text { if } x \bmod 2 R \in(R, 2 R), \\
0, & \text { if } x \bmod R=0,
\end{array} \text { for a.e. } t \in[0, T] .\right.
$$

As we can see from Lemma 1 the function $f^{*}$ is odd and $2 R$-periodic in the second variable. We consider an auxiliary differential equation

$$
y^{\prime \prime}=f^{*}(t, y) .
$$

Its purpose is cleared out in Lemma 2. By a solution of (3.4) we understand a function $y \in \operatorname{AC}^{1}([0, T])$ such that (3.4) is satisfied almost everywhere on $[0, T]$.

Lemma 2. If $y$ is a strictly monotone solution of equation (3.4) such that $y(0) \mathrm{mod}$ $R \neq 0, y(T) \bmod R \neq 0$, then the function

$$
x(t)=(\Delta \circ y)(t)=\left\{\begin{array}{ll}
y(t) \bmod 2 R & \text { if } y(t) \bmod 2 R \in[0, R), \\
2 R-(y(t) \bmod 2 R) & \text { if } y(t) \bmod 2 R \in[R, 2 R),
\end{array} \quad t \in[0, T]\right.
$$

is a solution of impulsive equation (1.1),(1.2) having exactly

$$
\left.\| \frac{y(0)}{R}\right\rfloor-\left\lfloor\frac{y(T)}{R}\right\rfloor
$$

impacts.

Proof. Let $y$ be a strictly increasing solution of (3.4). From Remark 3 it follows that $x$ is continuous on $[0, T]$ and $x(t) \in K$ for each $t \in[0, T]$. Let us denote

$$
k:=\left\lfloor\frac{y(0)}{R}\right\rfloor, \quad l:=\left\lfloor\frac{y(T)}{R}\right\rfloor, \quad p:=\left\lfloor\frac{y(0)}{R}\right\rfloor-\left\lfloor\frac{y(T)}{R}\right\rfloor .
$$

Then $p=|k-l|=l-k, k R<y(0)<(k+1) R, l R<y(T)<(l+1) R$. From the continuity and monotonicity it follows that there exist $0<t_{1}<t_{2}<\ldots<t_{p}<T$ such 
that

$$
y\left(t_{i}\right)=(k+i) R, \quad i=1, \ldots, p .
$$

Then for every $t \in[0, T]$ the equation $y(t) \bmod R=0$ is satisfied iff $t=t_{i}$ for some $i \in\{1, \ldots, p\}$, i.e. $x(t) \in\{0, R\}=\partial K$ iff $t=t_{i}$. This means that $x$ has exactly $p$ impacts.

Let us put $t_{0}=0, t_{p+1}=T$. For $i=0, \ldots, p, t \in\left(t_{i}, t_{i+1}\right)$ we have $y(t) \in$ $((k+i) R,(k+i+1) R)$ and according to Remark 3 we see that

$$
x^{\prime}(t)=\Delta^{\prime}(y(t)) y^{\prime}(t)=(-1)^{k+i} y^{\prime}(t) \quad t \in\left(t_{i}, t_{i+1}\right),
$$

i.e. $x^{\prime}$ is absolutely continuous on $\left(t_{i}, t_{i+1}\right)$. Moreover

$$
x^{\prime}\left(t_{i}+\right)=\lim _{t \rightarrow t_{i}+} x^{\prime}(t)=\lim _{t \rightarrow t_{i}+}(-1)^{k+i} y^{\prime}(t)=(-1)^{k+i} y^{\prime}\left(t_{i}\right), \quad i=0, \ldots, p
$$

and

$x^{\prime}\left(t_{i}-\right)=\lim _{t \rightarrow t_{i}-} x^{\prime}(t)=\lim _{t \rightarrow t_{i}-}(-1)^{k+i-1} y^{\prime}(t)=(-1)^{k+i-1} y^{\prime}\left(t_{i}\right), \quad i=1, \ldots, p+1$.

Therefore $\left.x\right|_{\left[t_{i}, t_{i+1}\right]}$ has absolutely continuous derivative on $\left[t_{i}, t_{i+1}\right], i=0, \ldots, p$ and $x$ satisfies (1.2).

Let $i \in\{0, \ldots, p\}$. If $y(t) \bmod 2 R \in(0, R)$ for each $t \in\left(t_{i}, t_{i+1}\right)$, then by (3.3), (3.5) we have

$$
x^{\prime \prime}(t)=(y(t) \bmod 2 R)^{\prime \prime}=y^{\prime \prime}(t)=f^{*}(t, y(t))=f(t, y(t) \bmod 2 R)=f(t, x(t))
$$

for a.e. $t \in\left(t_{i}, t_{i+1}\right)$. If $y(t) \bmod 2 R \in(R, 2 R)$ for $t \in\left(t_{i}, t_{i+1}\right)$, then

$$
\begin{aligned}
x^{\prime \prime}(t) & =(2 R-(y(t) \bmod 2 R))^{\prime \prime}=-y^{\prime \prime}(t)=-f^{*}(t, y(t)) \\
& =-(-f(t, 2 R-(y(t) \bmod 2 R)))=f(t, x(t))
\end{aligned}
$$

for a.e. $t \in\left(t_{i}, t_{i+1}\right)$. Therefore $x$ satisfies Eq. (1.1). The proof is similar for the case when $y$ is strictly decreasing.

Note that the function $f^{*}$ is not Carathéodory in general, since

$$
\lim _{x \rightarrow R k+} f^{*}(t, x)=-\lim _{x \rightarrow R k-} f^{*}(t, x) \in \mathbb{R} .
$$

for each $k \in \mathbb{Z}$ and a.e. $t \in[0, T]$, see Remark 2. Therefore we use regularization technique to obtain a solution of problem (3.4),(1.3), with $A, B \in \mathbb{R}$, satisfying $A$ mod $R \neq 0 \neq B \bmod R$.

For $n \in \mathbb{N}$, we put

$$
\eta_{n}(x)= \begin{cases}\frac{2 n}{R} x & \text { if } x \in\left[0, \frac{R}{2 n}\right), \\ 1 & \text { if } x \in\left[\frac{R}{2 n}, R\left(1-\frac{1}{2 n}\right)\right], \\ \frac{2 n}{R}(R-x) & \text { if } x \in\left(R\left(1-\frac{1}{2 n}\right), R\right] .\end{cases}
$$

Let us emphasize that for every $n \in \mathbb{N}$ the function $\eta_{n}$ has the following properties:

- $\eta_{n}:[0, R] \rightarrow \mathbb{R}$ is continuous, 
- $\eta_{n}(0)=\eta_{n}(R)=0$

- $0 \leq \eta_{n}(x) \leq 1$ for $x \in[0, R]$ and

- $\eta_{n}(x)=1$ for $x \in\left[\frac{R}{2 n}, R\left(1-\frac{1}{2 n}\right)\right]$.

Let us consider truncated functions

$$
f_{n}^{*}(t, x)=\eta_{n}(x \bmod R) f^{*}(t, x),
$$

for a.e. $t \in[0, T]$, all $x \in \mathbb{R}, n \in \mathbb{N}$.

Remark 4. Let us note that from the assumption (3.1), properties of $\eta_{n}$ and Remark 2 , it follows that $f_{n}^{*} \in \operatorname{Car}([0, T] \times \mathbb{R})$, and moreover $\left|f^{*}(t, x)\right| \leq m(t)$ as well as $\left|f_{n}^{*}(t, x)\right| \leq m(t)$ for a.e. $t \in[0, T]$, all $x \in \mathbb{R}$ and $n \in \mathbb{N}$.

To obtain the existence result for boundary value problem (3.4), (1.3) we consider auxiliary equation

for $n \in \mathbb{N}$.

$$
y^{\prime \prime}=f_{n}^{*}(t, y) \quad \text { for a.e. } t \in[0, T] .
$$

Lemma 3. Let us assume (3.1), (3.2), $A, B \in \mathbb{R}, A \bmod R \neq 0 \neq B \bmod R, n \in \mathbb{N}$. Then there exists at least one solution $y_{n} \in \mathrm{AC}^{1}([0, T])$ to the problem (3.7),(1.3) satisfying

$$
\left\|y_{n}\right\|_{\infty} \leq K, \quad\left|y_{n}^{\prime}(t)-\frac{B-A}{T}\right| \leq \bar{m}, t \in[0, T],
$$

where

$$
K:=|A|+|B|+T \bar{m} .
$$

Proof. Let us put

$$
G(t, s)= \begin{cases}\frac{t(s-T)}{T} & t \leq s, \\ \frac{s(t-T)}{T} & t>s,\end{cases}
$$

which is the Green's function of the boundary value problem

$$
y^{\prime \prime}=0 \quad \text { on }[0, T], \quad y(0)=0, \quad y(T)=0 .
$$

Let $T_{n}: \mathrm{C}^{1}([0, T]) \rightarrow \mathrm{C}^{1}([0, T])$ be an operator defined by

$$
T_{n} y(t)=\frac{t}{T} B+\frac{T-t}{T} A+\int_{0}^{T} G(t, s) f_{n}^{*}(s, y(s)) \mathrm{d} s, \quad y \in \mathrm{C}^{1}([0, T]),
$$

and a set

$$
\Omega=\left\{y \in \mathrm{C}^{1}([0, T]) \quad: \quad\|y\|_{\infty} \leq K, \quad\left\|y^{\prime}\right\|_{\infty} \leq K_{1}\right\}
$$

with (3.9) and $K_{1}:=\frac{|B-A|}{T}+\bar{m}$. According to Remark 4 and definition of $T_{n}$ we see that $T_{n}\left(\mathrm{C}^{1}([0, T])\right) \subset \Omega$. It is a standard argument to prove that $T_{n}$ are totally continuous on $\Omega$ - using Arzelà-Ascoli theorem. Therefore according to the Schauder fixed point theorem there exists a fixed point $y_{n} \in \Omega$ of $T_{n}$. Again, it is a standard procedure to prove that $y_{n}$ is a solution of (3.7),(1.3). 
Let us emphasize the fact that the upper estimates $K$ and $\bar{m}$ in (3.8) are independent of $n$.

Lemma 4. Let us assume (3.1), (3.2), $A, B \in \mathbb{R}, A \bmod R \neq 0 \neq B \bmod R$. If

$$
|B-A|>T \bar{m},
$$

then there exists a strictly monotone solution of problem (3.4), (1.3).

Proof. According to Lemma 3 for every $n \in \mathbb{N}$ there exists a solution $y_{n} \in$ $\mathrm{AC}^{1}([0, T])$ of problem (3.7), (1.3) satisfying (3.8), (3.9). Obviously, the sequences $\left\{y_{n}\right\}_{n=1}^{\infty}$ and $\left\{y_{n}^{\prime}\right\}_{n=1}^{\infty}$ are uniformly bounded. Moreover, due to (3.7) and Remark 4, for every $n \in \mathbb{N}$ and $t, s \in[0, T]$ we have

$$
\left|y_{n}^{\prime}(t)-y_{n}^{\prime}(s)\right|=\left|\int_{s}^{t} y_{n}^{\prime \prime}(\xi) \mathrm{d} \xi\right| \leq\left|\int_{s}^{t}\right| f_{n}^{*}\left(\xi, y_{n}(\xi)\right)|\mathrm{d} \xi| \leq\left|\int_{s}^{t} m(\xi) \mathrm{d} \xi\right| .
$$

Since $m \in \mathrm{L}^{1}([0, T])$, the sequence $\left\{y_{n}^{\prime}\right\}_{n=1}^{\infty}$ is equicontinuous. According to ArzelàAscoli theorem there exists a convergent (in $\left.\mathrm{C}^{1}([0, T])\right)$ subsequence $\left\{y_{k_{n}}\right\}_{n=1}^{\infty}$ and its limit $y \in \mathrm{C}^{1}([0, T])$. From (3.8) by taking limit $n \rightarrow \infty$ we have

$$
\frac{B-A}{T}-\bar{m} \leq y^{\prime}(t) \leq \frac{B-A}{T}+\bar{m}, \quad t \in[0, T] .
$$

From assumption (3.10) we see that $A \neq B$. There are two cases:

If $A<B$, then from (3.10) we get

$$
\frac{B-A}{T}>\bar{m}
$$

Consequently from the first inequality in (3.11) we see that $y^{\prime}>0$ on $[0, T]$, therefore $y$ is strictly increasing.

If $A>B$, then from (3.10) we have

$$
-\frac{B-A}{T}>\bar{m}
$$

Consequently from the second inequality in (3.11) we see that $y^{\prime}<0$ on $[0, T]$, therefore $y$ is strictly decreasing.

In both cases $y$ is a strictly monotone function. Since $y$ is strictly monotone and continuous, it follows that there exists a finite set $T_{y}$ such that for $t \in[0, T]$ the equality $y(t) \bmod R=0$ holds iff $t \in T_{y}$. If $T_{y}=\varnothing$, then we put $t_{0}=0, t_{1}=T$ and $p=0$. If $T_{y} \neq \varnothing$, we denote $T_{y}=\left\{t_{1}, t_{2}, \ldots, t_{p}\right\}$, where

$$
0=: t_{0}<t_{1}<t_{2}<\ldots<t_{p}<t_{p+1}:=T .
$$

Let $i \in\{0, \ldots, p\}$. Then for each $s_{1}, s_{2} \in\left(t_{i}, t_{i+1}\right), s_{1}<s_{2}$ we have

$$
\begin{gathered}
y^{\prime}\left(s_{2}\right)-y^{\prime}\left(s_{1}\right)=\lim _{n \rightarrow \infty}\left(y_{k_{n}}^{\prime}\left(s_{2}\right)-y_{k_{n}}^{\prime}\left(s_{1}\right)\right) \\
=\lim _{n \rightarrow \infty} \int_{s_{1}}^{s_{2}} y_{k_{n}}^{\prime \prime}(s) \mathrm{d} s=\lim _{n \rightarrow \infty} \int_{s_{1}}^{s_{2}} f_{k_{n}}^{*}\left(s, y_{k_{n}}(s)\right) \mathrm{d} s .
\end{gathered}
$$


Since $\left\{y_{k_{n}}\right\}_{n=1}^{\infty}$ uniformly converges to $y$ on $\left(t_{i}, t_{i+1}\right)$ and $y$ is strictly monotone and continuous on this interval, it follows that there exists $n_{0} \in \mathbb{N}$ such that for $n \geq n_{0}$ we have

$$
y_{k_{n}}(t) \bmod R \in\left[\frac{R}{2 k_{n}}, R\left(1-\frac{1}{2 k_{n}}\right)\right] \subset(0, R) \quad \text { for each } t \in\left(s_{1}, s_{2}\right) .
$$

Therefore by (3.6) and the last property of $\eta_{n}$ we get

$$
\lim _{n \rightarrow \infty} \int_{s_{1}}^{s_{2}} f_{k_{n}}^{*}\left(s, y_{k_{n}}(s)\right) \mathrm{d} s=\lim _{n \rightarrow \infty} \int_{s_{1}}^{s_{2}} f^{*}\left(s, y_{k_{n}}(s)\right) \mathrm{d} s=\int_{s_{1}}^{s_{2}} f^{*}(s, y(s)) \mathrm{d} s .
$$

Summarizing, we obtain

$$
y^{\prime}\left(s_{2}\right)-y^{\prime}\left(s_{1}\right)=\int_{s_{1}}^{s_{2}} f^{*}(s, y(s)) \mathrm{d} s
$$

for each $s_{1}, s_{2} \in\left(t_{i}, t_{i+1}\right)$. According to Remark 4 we see that $y \in \mathrm{AC}^{1}\left(\left[t_{i}, t_{i+1}\right]\right)$ and therefore $y \in \mathrm{AC}^{1}([0, T])$. Moreover $y$ satisfies differential equation (3.4) for a.e. $t \in[0, T]$. Obviously, $y$ satisfies (1.3), as well.

\section{MAIN RESUlts}

Now we are able to formulate and prove the existence theorems.

Theorem 2. Let (3.1) be satisfied, $A, B \in(0, R)$ and $p \in \mathbb{N}$ be such that

$$
p>\frac{T \bar{m}}{R}+1,
$$

where $\bar{m}$ is defined in (3.2). Then there exist at least two solutions of (1.1)-(1.3) having exactly p-impacts.

Proof. STEP 1. (Existence of solutions of auxiliary problems) For $i \in \mathbb{Z}$ satisfying $|i|=p$ we consider the differential equation (3.4) together with boundary conditions

$$
y(0)=A, \quad y(T)=i R+\theta_{i},
$$

where

$$
\theta_{i}= \begin{cases}B & \text { if } i \text { is even } \\ R-B & \text { if } i \text { is odd }\end{cases}
$$

Let us note that if $y$ satisfies (4.2), then

$$
\left.\left.\left.\| \frac{y(0)}{R}\right\rfloor-\left\lfloor\frac{y(T)}{R}\right\rfloor=\left\lfloor\frac{A}{R}\right\rfloor-\left\lfloor\frac{i R+\theta_{i}}{R}\right\rfloor=\| \frac{i R+\theta_{i}}{R}\right\rfloor=\| i+\frac{\theta_{i}}{R}\right\rfloor=|i| .
$$

According to (4.1), we have

$$
\left|i R+\theta_{i}-A\right| \geq|i R|-\left|\theta_{i}-A\right| \geq|i| R-R=(|i|-1) R=(p-1) R>T \bar{m},
$$


which is the assumption (3.10) for $B=i R+\theta_{i}$. Therefore we conclude that by Lemma 4 there exists at least one increasing solution $y_{1}$ of (3.4) with boundary condition

$$
y(0)=A, \quad y(T)=p R+\theta_{p},
$$

and at least one decreasing solution $y_{2}$ of (3.4) with boundary condition

$$
y(0)=A, \quad y(T)=-p R+\theta_{-p} .
$$

From (4.3) we get

$$
\|\left\lfloor\frac{y_{1}(0)}{R}\right\rfloor-\left\lfloor\frac{y_{1}(T)}{R}\right\rfloor=\left\lfloor\frac{y_{2}(0)}{R}\right\rfloor-\left\lfloor\frac{y_{2}(T)}{R}\right\rfloor=p .
$$

STEP 2. (Solutions of (1.1)-(1.3)) Let us define $x_{1}$ by (3.5) with $x_{1}$ and $y_{1}$ in place of $x$ and $y$, respectively. Then according to Lemma 2 and (4.4), the function $x_{1}$ is a solution of (1.1), (1.2) and it has exactly $p$ impacts. Moreover, $x_{1}(0)=y_{1}(0)=A$. It remains to prove that $y_{1}(T)=B$. If $p$ is even, then $y_{1}(T)=p R+B$. Obviously $y_{1}(T) \bmod 2 R=B \in(0, R)$ and then

$$
x_{1}(T)=y_{1}(T) \bmod 2 R=B .
$$

If $p$ is odd, then $y_{1}(T)=(p+1) R-B=(p-1) R+2 R-B$. Obviously $y_{1}(T)$ mod $2 R=2 R-B$ and then

$$
x_{1}(T)=2 R-\left(y_{1}(T) \bmod 2 R\right)=2 R-(2 R-B)=B .
$$

So the function $x_{1}$ is a solution of (1.1)-(1.3) having exactly $p$ impacts. Similarly, we define $x_{2}$ by (3.5) with $x_{2}$ and $y_{2}$ in place of $x$ and $y$, respectively, and prove that $x_{2}$ is solution of (1.1)-(1.3) having exactly $p$ impacts, in the same way. From the properties of $y_{i}$ we can see that $x_{1} \neq x_{2}$.

Corollary 1. Let (3.1) be satisfied, $A, B \in(0, R)$. Then there exist infinitely many solutions of (1.1)-(1.3).

Remark 5. Theorem 2 states that for $p$ satisfying (4.1) there are two solutions $x_{1}$, $x_{2}$ of (1.1)-(1.3). But from the proof we can say much more about these solutions. Let $t_{1}^{i}, \ldots, t_{p}^{i}$ be times of impacts of $x_{i}, t_{0}^{i}=0, t_{p+1}^{i}=T, i=1,2$. Then

$$
\begin{aligned}
& \text { - } x_{i}^{\prime}(t)(-1)^{i+j}>0 \text { for } t \in\left(t_{j-1}^{i}, t_{j}^{i}\right), j=1, \ldots, p+1, i=1,2, \\
& \text { - } x_{1}\left(t_{j}^{1}\right)=R \text { and } x_{2}\left(t_{j}^{2}\right)=0 \text { for odd } j \in\{1, \ldots, p\}, \\
& \text { - } x_{1}\left(t_{j}^{1}\right)=0 \text { and } x_{2}\left(t_{j}^{2}\right)=R \text { for even } j \in\{1, \ldots, p\} .
\end{aligned}
$$

For instance, this means that $x_{1}$ is increasing on the interval $\left[0, t_{1}^{1}\right]$, it hits $x=R$ at $t=t_{1}^{1}$, then it is decreasing until it is positive and so on.

The proof of Theorem 1 is almost a consequence of Theorem 2 . Proof. (Proof of Theorem 1) Let us put $\widetilde{R}:=b-a, \widetilde{K}=[0, \widetilde{R}]$ and

$$
\widetilde{f}(t, x):=f(t, x+a) \quad \text { a.e. } t \in[0, T] \text {, all } x \in[0, \widetilde{R}]=\widetilde{K},
$$


$\widetilde{A}:=A-a, \widetilde{B}:=B-a$. Then

$$
|\widetilde{f}(t, x)| \leq m(t) \text { for a.e. } t \in[0, T] \text {, all } x \in \widetilde{K} \text {. }
$$

According to Theorem 2 there exist two solutions $\widetilde{x}_{1}, \widetilde{x}_{2}$ of (1.1)-(1.3) with $f=\widetilde{f}$, $R=\widetilde{R}, K=\widetilde{K}, A=\widetilde{A}, B=\widetilde{B}$ having exactly $p$ impacts. It is not difficult to check that

$$
x_{1}(t):=\widetilde{x}_{1}(t)+a, \quad x_{2}(t):=\widetilde{x}_{2}(t)+a, \quad t \in[0, T]
$$

are solutions to problem (1.1)-(1.3) for $K=[a, b]$.

Example 1. Let us consider a one-dimensional billiard problem

$$
\begin{gathered}
y^{\prime \prime}=t^{\alpha}+\sqrt{|y|}, \quad \text { for a.e. } t \in[0, T], y(t) \in(-r, r), \\
y^{\prime}(s+)=-y^{\prime}(s-), \quad s \in(0, T),|y(s)|=r, \\
y(0)=y(T)=0,
\end{gathered}
$$

where $\alpha>0$. According to Theorem 1 putting $a=-r, b=r, A=0, B=0$, then for each $p \in \mathbb{N}$,

$$
p>\frac{T^{2}}{2 r}\left(\frac{T^{\alpha}}{\alpha+1}+\sqrt{r}\right)+1
$$

there exist at least two solutions of the Dirichlet problem having exactly $p$ impacts. Note that a similar result in [4] (Theorem 3.6) cannot be applied in this case, because the right-hand side of the differential equation is not Lipschitz-continuous in the second variable. Moreover, Theorem 1 gives more detailed multiplicity results than the result in [4].

Example 2. Let us consider oscillator

$$
y^{\prime \prime}=-\lambda y+q(t),
$$

with $\lambda>0, p$ the Lebesgue integrable function on $[0, T]$ with obstacles from above and from below, i.e. the solution has values in a compact interval $K=[-r, r]$. If the value reaches the boundary of $K$, then the impact occurs and the oscillator bounces back with absolutely elastic impact, which can be expressed by condition

$$
y^{\prime}(s+)=-y^{\prime}(s-), \quad \text { if }|y(s)|=r .
$$

Let us look for solutions of this equation with impact satisfying Dirichlet conditions $y(0)=A, y(T)=B$, where $|A|,|B|<r$. Since

$$
\max _{x \in[-r, r]}|-\lambda x+q(t)| \leq \lambda r+|q(t)|, \quad \text { for a.e. } t \in[0, T]
$$

we can conclude from Theorem 1 that for each integer $p$ greater than

$$
\frac{T}{2 r}\left(\lambda r T+\|q\|_{L^{1}}\right)+1,
$$

there exist at least two solutions of this boundary value problem with exactly $p$ impacts. 


\section{ACKNOWLEDGEMENT}

This work was supported by the grant No. 14-06958S of the Grant Agency of the Czech Republic.

\section{REFERENCES}

[1] M. Akhmet, Principles of Discontinuous Dynamical Systems. New York: Springer, 2010. doi: 10.1007/978-1-4419-6581-3.

[2] D. Bohneure and C. Fabry, "Periodic motions in impact oscillatiors with perfectly elastic bounces," Nonlinearity, vol. 15, pp. 1-17, 2002, doi: 10.1088/0951-7715/15/4/314.

[3] S. Deo and S. Pandit, Differential Systems Involving Impulses. Berlin: Springer-Verlag, 1982. doi: 10.1007/BFb0067476.

[4] G. Gabor, "On the Dirichlet problem in billiard spaces," J. Math. Anal. Appl., vol. 440, pp. 677691, 2016, doi: 10.1016/j.jmaa.2016.03.072.

[5] J. Graef, J. Henderson, and A. Ouahab, Impulsive Differential Inclusions. Berlin: Walter de Gruyter, 2013. doi: 10.1515/9783110295313.

[6] A. Halanai and D. Wexler, Qualitative Theory of Impulsive Systems. Moscow: Mir, 1971, in Russian.

[7] V. Kozlov and D. Treshchëv, Billiards: A Genetic Introduction to the Dynamics of Systems with Impacts, ser. Transl. Math. Monogr. Providence, RI: American Mathematical Society, 1991, vol. 89.

[8] V. Lakshmikantham, D. Bainov, and P. Simeonov, Theory of Impulsive Differential Equations, ser. Series in Modern Applied Mathematics. Singapore: World Scientific, 1989, vol. 6.

[9] D. Qian and P. J. Torres, "Periodic motions of linear impact oscillators via the successor map," SIAM J. Math. Anal., vol. 36, no. 6, pp. 1707-1725, 2002, doi: 10.1137/S003614100343771X.

[10] Rachunkova and Tomecek, State-Dependent Impulses. Boundary Value Problems on Compact Interval, ser. Atlantis Briefs in Differential Equations. Atlantis Press, 2015, vol. 6, doi: 10.2991/978-94-6239-127-7.

[11] A. Samoilenko and N. Perestyuk, Impulsive Differential Equations. Singapore: World Scientific, 1995.

\section{Author's address}

\section{Jan Tomeček}

Department of Mathematical Analysis, and Applications of Mathematics,, Faculty of Science, Palacký University,, 17. listopadu 12, 77146 Olomouc, Czechia

E-mail address: jan.tomecek@upol.cz 